\title{
Tibanna: software for scalable execution of porta- ble pipelines on the cloud
}

\author{
Soohyun Lee ${ }^{1}$, Jeremy Johnson ${ }^{1}$, Carl Vitzthum, Koray Kırlı, Burak H. Alver ${ }^{*}$, Peter J. Park ${ }^{*}$ \\ 1 Equally contributed \\ Department of Biomedical Informatics, Harvard Medical School, Boston, MA, USA. \\ *To whom correspondence should be addressed.
}

\begin{abstract}
Summary: We introduce Tibanna, an open-source software tool for automated execution of bioinformatics pipelines on Amazon Web Services (AWS). Tibanna accepts reproducible and portable pipeline standards including Common Workflow Language (CWL), Workflow Description Language (WDL) and Docker. It adopts a strategy of isolation and optimization of individual executions, combined with a serverless scheduling approach. Pipelines are executed and monitored using local commands or the Python Application Programming Interface (API) and cloud configuration is automatically handled. Tibanna is well suited for projects with a range of computational requirements, including those with large and widely fluctuating loads. Notably, it has been used to process terabytes of data for the 4D Nucleome (4DN) Network.
\end{abstract}

Availability: Source code is available on GitHub at https://github.com/4dn-dcic/tibanna.

Contact: peter_park@hms.harvard.edu

\section{Introduction}

Efficient execution of data processing and analysis pipelines is essential in many areas of research that involve large datasets. However, integration of a pipeline with local computing infrastructure is often a painstaking process. The need for such integration leads to redundant creation of seemingly identical pipelines, decreasing reproducibility and efficiency.

Cloud platforms present a number of advantages for large-scale data analysis, including scalability and efficient data sharing. However, the frequent lack of separation between workflow specifications and platformspecific configuration impedes transition to the cloud. Having to handle detailed aspects of the cloud platform renders the advantages of cloud computing impractical for many pipeline developers.

For effective separation of pipelines from platforms, pipelines should be made portable and standardized, and all platform-specific tasks must be delegated to a separate pipeline management tool. To enable this framework, there have been efforts to create a standard for portable pipelines. Common Workflow Language (CWL) (https://www.commonwl.org/) and Workflow Description Language (WDL) (https://software.broadinstitute.org/wdl/) describe the structure of a pipeline (e.g., steps, inputs and outputs), whereas Docker and Singularity (Kurtzner et al., 2017) enable generation of portable images of executable components and dependencies. Support for standard pipeline languages is becoming more widely adopted. In addition, Galaxy (Giardine et al. 2005), a GUI-based bioinformatics analysis platform, is planned to support CWL in the near future. Domain Specific Languages (DSLs) such as Nextflow (Tommaso et al., 2017) or Snakemake (Köster and Rahmann 2012) provide better expressive power than CWL, but are bound to a single pipeline manager.

At the Data Integration and Coordination Center (DCIC) for the NIHsponsored 4D Nucleome (4DN) Network (Dekker at al. 2017), our aim is to efficiently process a large number of datasets from multiple experimental types submitted by member laboratories. We therefore require a workflow management system that supports standardized and portable pipelines (CWL/WDL) on a public platform. We chose Amazon Web Services (AWS), the most widely used commercial cloud service. Additionally, the Network needs an open-source implementation so the scientific community can easily run the same $4 \mathrm{DN}$ pipelines on its own data.

\section{Results}

We have developed and used Tibanna as an open-source pipeline manager and automated cloud resource allocator for running Docker-based pipelines on AWS. Tibanna integrates easily with other systems through its Python API.

\subsection{Overview}

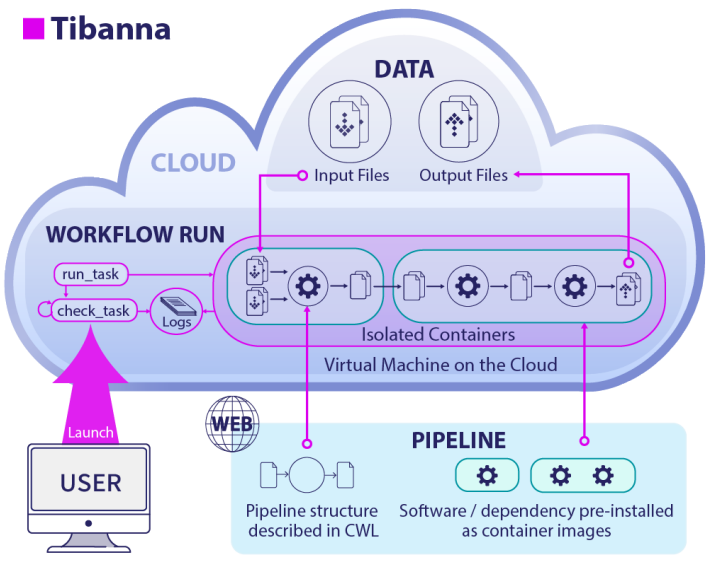

Fig 1. Tibanna. For each pipeline execution, Tibanna creates a workflow run by launching a customized virtual machine (EC2), runs the pipeline and destroys itself when finished. Scheduling is performed individually by serverless components. 


\section{S. Lee et al.}

Tibanna creates an instance of an individually pre-configured virtual machine (EC2) for each job (Figure 1). The instance autonomously fetches data and the pipeline, runs the pipeline, stores logs and output files on the cloud and finally, terminates itself. Instead of depending on a master server or queue, each job gets its own serverless scheduler comprised of AWS Lambdas coordinated by the AWS Step Function. The 4DN DCIC uses additional Lambdas to communicate with our Data Portal.

\subsection{Execution unit}

Tibanna runs a single execution unit, and dependencies can be managed through its dependency feature. This allows one to decide and manage execution units based on resource requirements and logical grouping, and determine what intermediate files to keep for future re-processing and curation. An execution unit may be a whole pipeline, a sub-pipeline or a single step, and these units are not defined by the pipeline structure itself. We found that tools that attempt to auto-determine execution units tend to make non-optimal choices and are difficult to integrate with desired execution designs.

\subsection{How to use Tibanna}

Tibanna auto-configures cloud components and permissions with a single command. Its only requirements are to provide input data on the cloud and to prepare a publicly available pipeline in CWL or WDL, along with a public Docker image. Each job is described as a JSON file or a Python dictionary object, specifying the pipeline, input files and parameters, and where the output should be collected. Submission and monitoring of jobs is performed locally from the command line or using the Python API. Logs can also be retrieved similarly or through the AWS Web Console. A user with a private key file can securely connect to running instances for more detailed monitoring. An easy-to-follow documentation comes with the software for more details.

\subsection{Execution example}

In addition to running pipelines for $4 \mathrm{DN}$, Tibanna has been used by external users to call transposon insertions from 1,000 30X whole genome sequencing data sets. 1,000 jobs were simultaneously executed, each running for 4-8 hours on a spot instance with 16 cores and 32GB memory and $250 \mathrm{~GB}$ disk size, costing about $\$ 1.6$ per run.

\subsection{Comparison to other tools}

Tibanna offers several advantages compared to existing workflow management tools (Supplementary Table 1), specifically for our need to automatically execute and monitor various portable pipelines in integration with a data portal.

Local CWL executors (e.g. Cwltool, Rabix (Kaushik 2016) and CWLAirflow (Kotliar et al. 2017)) do not perform resource allocation. Snakemake recently started supporting local CWL executions with cloud storage but also does not handle resource management on AWS.

Tools that perform cloud resource allocation uses one of the three approaches; AWS Batch, a resizable cluster, or individual customization.

(1) AWS Batch AWS Batch (https://aws.amazon.com/batch/) is an AWS service to run batch jobs, but it is not easy to be used directly with CWL/Docker, often exhibits a mysteriously long wait time, and lacks many functionalities. Examples of tools based on AWS Batch include Nextflow, Funnel (https://ohsu-comp-bio.github.io/funnel/) and Cromwell. Nextflow supports Docker, but using CWL requires conversion to the Nextflow format and the converter is not functional at this point.

Cromwell (https://software.broadinstitute.org/wdl/) was originally developed to run WDL on Google Cloud Platform (GCP), although it now supports CWL and AWS through AWS Batch. However, it leaves all the cloud configuration to the user, and due to the lack of an API, a large-scale batch run requires a cumbersome generation of many input JSON files and manual tracing of job IDs for proper output matching. Monitoring functionality that is critical for large-scale projects is not provided by either Cromwell or AWS Batch. Funnel also requires users to build a custom Amazon Machine Image (AMI).

(2) Resizable cluster StarCluster (http://star.mit.edu/cluster/docs/latest), an older tool without CWL/Docker support, and Toil (Vivian et al., 2017) work by creating a resizable cluster of EC2 instances of the same CPU and memory and an additional master instance. They require extra work for configuring and managing clusters. Nextflow also uses an auto-scalable cluster as an alternative. Arvados (https://arvados.org/) supports CWL and Docker and involves a cluster of dynamically allocated cloud instances where jobs are dispatched by SLURM. However, the initial setup process is quite complicated. Arvados also uses its own storage organization which required additional development to access and is not compatible with the file organizations used by our portal.

(3) Individual customization Tibanna instead creates a specific compute environment for an individual execution at launch, and removes it when the job is done. This approach is also adopted by Seven Bridges (https://www.sevenbridges.com/) and DNANexus (https://www.dnanexus.com/), which, as commercial portal services, also offer full automation but charge extra fees and do not easily integrate with other systems.

At 4DN DCIC, we receive data sets that vary in input file size, with vastly different memory and storage requirements. For example, the contact matrix generation sub-workflow of the $4 \mathrm{DN}$ Hi-C data processing pipeline involves in-memory generation of a matrix that represents nonzero interactions of all genomic locations. As the size of input datasets (i.e., reads representing contacts) varies $>10$-fold (up to $76 \mathrm{~Gb}$ ), memory requirement also varies substantially, thus necessitating machines with different memory sizes for efficient computation. For this level of variability at the execution level, individual customization makes better use of the elastic compute environment of the cloud compared to cluster allocation.

\section{Conclusion}

Tibanna is a pipeline management system for the AWS cloud. Tibanna has been used to process $4 \mathrm{DN}$ data. We believe Tibanna will be useful for many other AWS projects.

\section{Acknowledgements}

We thank the members of the 4DN DCIC, the Park lab and the Avillach lab at the Department of Biomedical Informatics at Harvard Medical School for valuable feedback and the E. Alice Lee lab at Boston Children's Hospital for beta-testing Tibanna. We are especially thankful to Andy Schroeder, Peter Kerpedjiev, Martin Owens and Chuck McCallum for code review/contribution, Shannon Ehmsen for preparing the figure, and Alon Galor, Dhawal Jain, Su Wang, Geoff Nelson and Marc Rubenfield (Veritas Genetics) for comments on the manuscript.

\section{Funding}

This work has been supported by the National Institutes of Health grant 5U01CA200059 awarded to PJP.

Conflict of Interest: none declared. 
bioRxiv preprint doi: https://doi.org/10.1101/440974; this version posted April 29, 2019. The copyright holder for this preprint (which was not certified by peer review) is the author/funder, who has granted bioRxiv a license to display the preprint in perpetuity. It is made available under aCC-BY-NC-ND 4.0 International license.

\section{Article short title}

\section{References}

Dekker,J et al. (2017) "The 4D nucleome project, Nature, 549: 219-226.

Gaurav,K (2016) Rabix: an open-source workflow executor supporting recomputability and interoperability of workflow descriptions, Pac Symp Biocomput. 22:154-165.

Giardine,B et al. (2005) Galaxy: A platform for interactive large-scale genome analysis, Genome Res 15(10): 1451-1455.

Köster,J and Rahmann,S. (2012) Snakemake — a scalable bioinformatics workflow engine. Bioinformatics, 28(19): 2520-2522
Kotliar,M et al. (2017) CWL-Airflow: a lightweight pipeline manager supporting Common Workflow Language, bioRxiv 249243

Kurtzner,GM et al. (2017) Singularity: Scientific containers for mobility of compute, PLoS ONE, 12(5): e0177459

Tommaso,PD et al. (2017) Nextflow enables reproducible computational workflows, Nat Biotechnol 35:316-319

Vivian,J et al. (2017) Toil enables reproducible, open source, big biomedical data analyses, Nat Biotechnol 35:314-316 
bioRxiv preprint doi: https://doi.org/10 1101/440974; this version posted April 29,2019 . The copyright holder for this preprint (which was not certified by peer review) is the author/funder, who has granted bioRxiv a license to display the preprint in perpetuity. It is made available under aCC-BY-NC-ND 4.0 International license.

\section{Supplementary Table 1. Comparison of cloud-friendly pipeline executors}

Cloud-friendly pipeline executors are compared in various aspects, focused on compatibility with portable languages and degree of support for AWS cloud.

\begin{tabular}{|c|c|c|c|c|c|c|c|c|}
\hline & Tibanna & Seven Bridges & DNA Nexus & Nextflow & Toil & Arvados & Cromwell & Snakemake \\
\hline $\begin{array}{l}\text { Common Workflow Language (CWL) } \\
\text { support }\end{array}$ & yes & yes & no & no & yes & yes & yes & yes \\
\hline $\begin{array}{l}\text { Workflow Description Language } \\
\text { (WDL) support }\end{array}$ & yes & no & yes & no & yes & no & yes & no \\
\hline $\begin{array}{l}\text { computing on Amazon Web Services } \\
\text { (AWS) }\end{array}$ & yes & yes & yes & yes & yes & yes & yes & no \\
\hline $\begin{array}{l}\text { computing on Google Cloud Platform } \\
\text { (GCP) }\end{array}$ & no & yes & no & yes & yes & yes & yes & yes \\
\hline $\begin{array}{l}\text { easy to integrate with user's own } \\
\text { cloud data storage system }\end{array}$ & yes & moderate & no & yes & yes & no & yes & yes \\
\hline free and open source & yes & no & no & yes & yes & yes & yes & yes \\
\hline Graphic User Interface (GUI) & via AWS & yes & yes & no & no & yes & no & no \\
\hline Command-line interface (CLI) & yes & yes & yes & yes & yes & yes & yes & yes \\
\hline $\begin{array}{l}\text { Python Application Programming In- } \\
\text { terface (API) }\end{array}$ & yes & yes & yes & no & yes & yes & no & yes \\
\hline easy setup on AWS cloud & yes & yes & yes & no & no & no & no & - \\
\hline $\begin{array}{l}\text { cloud resource allocation approach } \\
\text { on AWS }\end{array}$ & individual & individual & individual & $\begin{array}{l}\text { cluster } \\
\text { AWS Batch }\end{array}$ & cluster & cluster & AWS Batch & - \\
\hline master server required & no & no & no & yes & yes & yes & no & no \\
\hline Comes with its own portal service & no & yes & yes & no & no & yes & no & no \\
\hline
\end{tabular}

